\title{
PREDICTIVE SUCCESS FACTORS FOR CT-GUIDED FINE NEEDLE ASPIRATION BIOPSY OF PULMONARY LESIONS
}

\author{
Marcos Duarte Guimarães, ${ }^{I}$ Rubens Chojniak, Jefferson L Gross, ${ }^{\text {II }}$ Almir G.V. \\ Bitencourt ${ }^{\mathrm{I}}$
}

doi: $10.1590 / \mathbf{S 1 8 0 7 - 5 9 3 2 2 0 0 9 0 0 1 2 0 0 0 0 2}$

Guimarães MD, Chojniak R, Gross JL, Bitencourt AGV. Predictive success factors for ct-guided fine needle aspiration biopsy of pulmonary lesions. Clinics. 2009;64(12):1139-44.

OBJECTIVE: Computed tomography-guided percutaneous fine needle aspiration biopsy of lung lesions is a simple, safe and reproducible procedure. Currently, it is widely used to diagnose lung lesions. However, different factors can influence the success rates of this procedure. The purpose of this study was to determine the influence of radiological and procedural characteristics in predicting the success rates of computed tomography-guided fine needle aspiration biopsy of lung lesions.

SUBJECTS AND METHODS: A retrospective study was developed and involved 340 patients who were submitted to a consecutive series of 362 computed tomography-guided fine needle aspiration biopsies of lung lesions, between July 1996 and June 2004 , using 22-gauge needles (Chiba). Variables such as the radiological characteristics of the lesions, secondary pulmonary radiological findings, and procedural techniques were studied.

RESULTS: For this study, 304 (84\%) fine needle aspiration biopsies of lung lesions provided sufficient material for cytological evaluation. The variables that predicted sufficient material for cytological evaluation were lesions larger than $40 \mathrm{~mm}(\mathrm{p}=0.02)$, lesions on the superior lung lobes $(\mathrm{p}=0.02)$, and suspicion of primary lung malignancy $(\mathrm{p}=0.03)$. From the multivariate analysis, the only predictive variable for success of the biopsies was localization on the superior lobes $(\mathrm{p}=0.01)$.

CONCLUSIONS: Computed tomography-guided percutaneous fine needle aspiration biopsy of lung lesions showed greater rates of success in biopsies performed in patients with suspicion of primary lung malignancy, with lesions located in the superior lobes, and that have diameters equal to and larger than $40 \mathrm{~mm}$.

KEYWORDS: Lung neoplasms. Computed tomography. Fine needle aspiration biopsy.

\section{INTRODUCTION}

Computed tomography (CT)-guided percutaneous fine needle aspiration biopsy (FNAB) of lung lesions is currently one of the most commonly used techniques to determine the nature of lung lesions, such as nodules and tumors. ${ }^{1}$ Because it is a simple, safe and reproducible technique with lower costs than a surgical biopsy, it has been widely accepted..$^{2-4}$

Significant variations in the accuracy of the procedure

${ }^{\text {I }}$ Department of Diagnostic Imaging, Hospital A C Camargo - São Paulo / SP, Brazil.

II Thoracic Surgery, Hospital A C Camargo - São Paulo/SP, Brazil.

Email: marcosduarte500@yahoo.com.br

Tel: 55112189.5000

Received for publication on July 22, 2009

Accepted for publication on August 11, 2009 have been reported in the literature. Some studies had over $90 \%$ accuracy. However, very few studies have examined the influence of the radiological characteristics of pulmonary lesions on the results of CT-guided FNAB. ${ }^{5-7}$

The purpose of this study was to evaluate the influence of radiological and procedural characteristics in predicting the success rates of CT-guided FNAB of lung lesions.

\section{SUBJECTS AND METHODS}

This is a retrospective analysis of all consecutive patients who were received CT-guided FNAB of pulmonary lesions at an oncological center in Brazil between 1996 and 2004.

From a total of 491 procedures evaluated, 362 (73.7\%) were considered eligible for this study. Of the 129 excluded procedures, $6(1.2 \%)$ were excluded because the lesions 
were defined as being on the chest wall or pleura after cytological evaluation or on follow-up; 26 (5.3\%) were excluded for having divergences between the admission and chart registrations; and 97 (19.8\%) were excluded because the procedure was performed with cutting needles, which was not the subject of this study. A total of 362 procedures performed on 340 patients are reported. Of these 362 procedures, 319 were of lesions submitted to biopsy once; 20 were of lesions submitted to biopsy twice; and one was of a lesion submitted to biopsy three times. Written informed consent was obtained from all patients.

Information was collected from the charts available at the hospital's Medical Archive Service (MAS) and the percutaneous biopsy forms. These forms contained data regarding the location, size of the lesions, distance between the lesion and the needle entrance site, the number of lesions, contour aspects of the lesions, and the relationship of the contour aspects to the adjacent structures such as the mediastinum or chest wall. Secondary radiological findings such as atelectasis, pleural effusion, cavitations, necrosis, infiltrates, adenopathy and the presence of other tumors were also included. Radiologic findings were based on the CT report prior to the procedure.

The FNABs were conducted by an oncologic radiologist with over ten years experience in CT-guided FNAB or by a resident in radiology under his guidance, following the standard procedure defined by the Imaging Department. ${ }^{8}$

At the Imaging Department, coagulation tests were routinely checked. When these tests were within normal parameters, the biopsy was planned and performed using a chest $\mathrm{CT}$ acquired with thin slices (3-5 $\mathrm{mm}$ ). The computer's cursor was used to measure the size of the lesions and the distances of the lesions from the biopsy needle entrance site. The skin was prepped with an antiseptic solution, and local anesthesia with $1 \%$ lidocaine was applied. The punction needle was introduced, and new tomographic cuts were obtained in order to confirm or modify the needle position. The biopsies were performed within a breath-hold.

The FNABs were performed using 22-gauge "CHIBA" type needles; subsequently, the obtained material was prepared in smears and immersed in $90 \%$ alcohol. Due to the retrospective nature of our study, it was not possible to determine the number of thoracic transfixations done by the FNAB procedure. However, the number of thoracic transfixations was indirectly obtained using the number of smears made by the puncture. In the experience of the Department of Imaging at the Hospital of Cancer, each puncture made around four smears.

The collected material was sent to the Department of Pathological Anatomy for analysis, where it was stained with hematoxylin-eosin (HE) and classified as adequate or inadequate for analysis, following department policy. When a sample was considered adequate by the pathologist, it was further categorized as positive, negative or suspicious for malignancy, and a specific diagnosis was provided when possible. For this study, we defined the FNAB as a success when the samples obtained were adequate for analysis by the pathologist.

The possible results included the following: malignant, when a specific type of cancer could be recognized; suspicion of malignancy, when atypical cells were present but were insufficient for a definitive diagnosis; negative for malignancy, when the sample was representative and without atypical cells; and possibly benign, when a benign tumor, inflammatory process or infection could be recognized.

By correlating the patient radiological data with the sample analysis results and complications, we were able to determine the predictive success rates of CT-guided FNAB of lung lesions. Descriptive statistics were used when applied. The chi-square test or Fisher's exact test was applied when appropriate. A $P$ value was considered statistically significant when equal to or less than 0.05 . The multivariable model of logistic regression analysis was applied using the variables that had $\mathrm{p}<0.05$ in univariate analysis; this model was used to identify possible predictive factors for the success of CT-guided FNAB.

\section{RESULTS}

From the 362 FNABs performed, $212(58.6 \%)$ were performed on male patients, and $150(41.4 \%)$ were performed on female patients. The group mean age was 61 \pm 16 years, and the median age was 63 years old. The reason for the FNAB referral was to obtain a primary diagnosis of a focal lesion suspected to be malignant in 215 (59.4\%) procedures and to document possible secondary malignancy in $147(40.6 \%)$ procedures.

The lung lesions were right-sided in 163 (45\%) procedures and left-sided in $154(42.5 \%)$ procedures. The lesion distribution by pulmonary lobes was as follows: $76(21.0 \%)$ on the left superior lobe, $60(16.6 \%)$ on the right superior lobe, $58(16.0 \%)$ on the right inferior lobe, $48(13.3 \%)$ on the left inferior lobe, and $27(7.5 \%)$ on the middle lobe. In addition, this information was not available in 93 cases $(25.7 \%)$.

The diameter of the lung lesions varied from 9 to 140 $\mathrm{mm}$ (mean: $51.5 \pm 24.3 \mathrm{~mm}$; median: $40 \mathrm{~mm}$ ). The distance between the lesion and the biopsy entry point on the skin varied from 10 to $130 \mathrm{~mm}$ (mean: $44 \pm 20.9 \mathrm{~mm}$; median: $52 \mathrm{~mm})$. Additionally, $71(40.1 \%)$ lesions were in contact with the pleura, and 106 (59.9\%) had pulmonary tissue positioned between the lesions and the pleura. The lesion 
contour type was irregular in 96 patients $(26.5 \%)$, spiculated in 38 patients $(10.5 \%)$, smooth in 37 patients $(10.2 \%)$, and lobulated in 22 patients $(6.0 \%)$. This information was not available in 169 cases (46.8\%). In $148(40.9 \%)$ of the procedures, the patient had one lung lesion, and in $25(6.9 \%)$ of the procedures, the patient had two lesions. In $22(6.1 \%)$ of the procedures, the patients had three lesions, and in 43 $(11.9 \%)$ of the procedures, four or more lung lesions were present.

The most common secondary radiologic findings were adenopathy (34 patients; 9.4\%), additional tumor (32 patients; $8.8 \%$ ), cavitation (16 patients; $4.4 \%$ ), necrosis (14 patients; 3.9\%), infiltration (13 patients; 3.3\%), pleural effusion (10 patients; $2.8 \%$ ), and opacification on the present lesion (four patients; $1.1 \%$ ).

From a total of 362 needle biopsies, a cytological result was obtained from the patients' charts for 357 (98.5\%). The success rate for obtaining adequate material for cytological analysis is shown in table 1 .

Table 1 - Cytological analysis results of 357 FNABs

\begin{tabular}{lcc}
\hline Material & $\mathrm{n}$ & $(\%)$ \\
\hline Adequate for Analysis & 204 & 56.4 \\
Malignant & 38 & 10.5 \\
Suspicion of malignancy & 45 & 12.4 \\
Negative for malignancy & 17 & 4.7 \\
Possibly Benign & 304 & 84.0 \\
Total & & \\
Inadequate for Analysis & 44 & 12.1 \\
Insufficient & 9 & 2.5 \\
Inadequate & 53 & 14.6 \\
Total & 5 & 1.4 \\
No information & 362 & 100 \\
Total & &
\end{tabular}

Of the 357 punctions evaluated by the pathologist, the material was considered adequate for analysis in 304 biopsies (84\%) and inadequate for analysis in 53 biopsies $(14.6 \%)$.

The number of smears obtained per procedure varied from one to thirty-six (mean \pm SD: $8.4 \pm 5.7$ median: 7.0). Procedures that provided more than four smears had a proportionately larger amount of adequate material for analysis $(88.1 \%$ vs. $78.5 \%$; $\mathrm{p}=0.04)$.

The proportion of biopsies with material judged adequate for analysis was higher in the patients referred for lesions suspected to be primary malignancy, as compared to the patients referred for lung lesions suspected to be secondary malignancy (Table 2). There was no difference in the material adequacy rate in patients of different gender or age groups (Table 2).

Table 3 shows the distribution of the radiological characteristics of the lesions and the corresponding rates of cytological material adequacy. The frequency of adequate material was higher in the lesions located in the superior lobes, as compared with the middle and inferior lobes. The larger lesion diameters $(\geq 40 \mathrm{~mm})$ also resulted in higher rates of success in obtaining adequate cytological material (Table 3). There was no statistical difference in material adequacy rates for series of biopsies performed in groups of patients with different secondary radiological lung findings. The total number of smears was not predictive of biopsy success.

In the multivariate analysis model, the only predictive variable for success of the biopsies was localization on the superior lobes (Table 4).

\section{DISCUSSION}

The overall success rate in our study was $84 \%$, which is similar to other studies of FNAB of lung lesions without

Table 2 - Material adequacy according to patient gender and age

\begin{tabular}{lccc}
\hline Variables & $\boldsymbol{n}(\boldsymbol{\%})$ & Adequate Material $\boldsymbol{n}(\boldsymbol{\%})$ & Inadequate Material $\boldsymbol{n}(\boldsymbol{\%})$ \\
\hline Gender & & & $\boldsymbol{p}$ \\
$\quad$ Male & $208(58.2)$ & $178(85.6)$ & $30(14.4)$ \\
$\quad$ Female & $149(41.8)$ & $126(84.6)$ & $23(15.4)$ \\
Age (mean) & & & \\
$\quad<63$ years & $120(48.8)$ & $97(80.8)$ & $23(19.2)$ \\
$\quad \geq 63$ years & $126(51.2)$ & $112(88.9)$ & $14(11.1)$ \\
Clinical Suspicion & & & 0.07 \\
Primary malignancy & $211(59.1)$ & $187(89.0)$ & $24(11.0)$ \\
Secondary malignancy* & $146(40.9)$ & $117(80.1)$ & $29(19.9)$ \\
\hline
\end{tabular}

*Secondary malignancy - suspicion of metastatic disease related to a known neoplasm. 
Table 3 - Analysis of the radiological characteristics of the lung lesions in relation to the adequate and inadequate material

\begin{tabular}{|c|c|c|c|c|}
\hline Variables & $n(\%)$ & $\begin{array}{c}\text { Adequate Material } \\
n(\%)\end{array}$ & $\begin{array}{c}\text { Inadequate Material } \\
n(\%)\end{array}$ & $p$ \\
\hline Contours & & & & 0.63 \\
\hline Smooth & 37 (19.2) & $31(83.8)$ & $6(16.2)$ & \\
\hline Lobulated & $22(11.4)$ & $21(95.5)$ & $1(4.5)$ & \\
\hline Spiculated & $38(19.7)$ & $33(86.8)$ & $5(13.2)$ & \\
\hline Irreg. non-specif. ${ }^{* 1}$ & $96(49.7)$ & $83(86.5)$ & $13(13.5)$ & \\
\hline Width $^{* 2}$ & & & & 0.23 \\
\hline$\leq 52 \mathrm{~mm}$ & $112(49.3)$ & $92(82.1)$ & $20(17.9)$ & \\
\hline$>52 \mathrm{~mm}$ & $115(50.7)$ & $101(87.8)$ & $14(12.2)$ & \\
\hline \multicolumn{5}{|l|}{ Lung } \\
\hline Right & $161(51.4)$ & $138(85.7)$ & $23(14.3)$ & 0.49 \\
\hline Left & $152(48.6)$ & $126(82.9)$ & $26(17.1)$ & \\
\hline \multicolumn{5}{|l|}{$\operatorname{Size}^{* 3}$} \\
\hline$<40 \mathrm{~mm}$ & $138(49.5)$ & $110(79.7)$ & $28(20.3)$ & 0.02 \\
\hline$\geq 40 \mathrm{~mm}$ & $141(50.5)$ & $127(90.1)$ & $14(9.9)$ & \\
\hline \multicolumn{5}{|l|}{ No. of Lesions } \\
\hline One & $148(62.1)$ & $125(85.0)$ & $22(15.0)$ & 0.29 \\
\hline Two & $25(10.5)$ & $19(76.0)$ & $6(24.0)$ & \\
\hline Three & $22(9.2)$ & $21(95.5)$ & $1(4.5)$ & \\
\hline Four or more & $43(18.2)$ & $35(81.4)$ & $8(18.6)$ & \\
\hline \multicolumn{5}{|l|}{ Lobes } \\
\hline Superior & $133(49.5)$ & $121(91.0)$ & $12(9.0)$ & 0.02 \\
\hline Middle & $30(11.1)$ & $23(76.7)$ & $7(23.3)$ & \\
\hline Inferior & $106(39.4)$ & $84(79.2)$ & $22(20.8)$ & \\
\hline Pleura Relation & & & & 0.49 \\
\hline Yes & $71(40.1)$ & $61(85.9)$ & $10(14.1)$ & \\
\hline No & $106(59.9)$ & $87(82.0)$ & $19(18)$ & \\
\hline Vital Organs ${ }^{* 4}$ & & & & 0.20 \\
\hline Yes & $33(18.6)$ & 30 (90.9) & $3(9.1)$ & \\
\hline No & $144(81.4)$ & 118 (84.9) & $26(18.1)$ & \\
\hline
\end{tabular}

*1 Irreg. non-specif., when the contour of the pulmonary injury was classified as irregular by the radiologist without other specifications.

*2 Superficial, when less than or equal to medium size; deep, when larger than medium size.

*3 Smaller Size, when less than or equal to medium size; larger size, when larger than medium size.

*4 Vital Organs, when there is a relation to structures considered as vital: aorta, main bronchi, heart, pulmonary hilum, trachea, pericardium, inferior vena cava, and pulmonary arteries and veins.

Table 4 - Multivariate model analysis for success in patients undergoing CT-guided FNAB of lung lesions

\begin{tabular}{lcccc}
\hline Variables & \multicolumn{3}{c}{ 95\% Confidence Interval } & \multirow{p}{*}{ Superior Limit } \\
\cline { 2 - 5 } & Odds Ratio & Inferior Limit & 4.55 & 0.10 \\
\hline Primary Diagnosis & 1.99 & 0.87 & 8.35 & $<.01$ \\
Localization & 3.37 & 1.36 & 1.01 & 0.05 \\
Size & 0.44 & 0.19 & 1.90 & 0.59 \\
Number of Smears & 0.79 & 0.33 & & \\
\hline
\end{tabular}


immediate cytological examination. ${ }^{9-13}$ On univariate analysis, procedures that were able to provide more than four smears obtained a larger amount of adequate material for analysis when compared to the procedures that provided less than or equal to four smears. These results tend to support the recommendation of more than one chest transfixation by the FNAB procedure for thoracic lesions. Regarding the radiological characteristics, the lesions larger than $40 \mathrm{~mm}$, those located in the superior lobes and suspicion of primary malignancy were predictive of the FNAB success.

Layfield et al. (1996) demonstrated that the localization and size of thoracic injuries affect the diagnostic accuracy of FNAB of the thorax, with the best results in peripheral and larger lesions. ${ }^{5}$ Yankelevitz et al. (1997), when studying small lesions (less or equal to $30 \mathrm{~mm}$ ), demonstrated that the distance between the nodule and the pleural surface did not influence the biopsy accuracy. ${ }^{6}$ In our study, there was also no difference in obtaining adequate material for analysis between the superficial and deep lesions.

Miller et al. (1998) demonstrated that spiculated contours and deep seated lesions were predictive characteristics of success in obtaining adequate material for analysis and diagnosis. ${ }^{7}$ In this study, we did not observe this relationship.

Many authors have found larger amounts of adequate material for analysis and greater accuracy in the lesions that were considered large (greater than $1.5 \mathrm{~cm}$ ) as compared with the lesions that were considered small..$^{3,11,13-16}$ In this study, lesions with diameters equal to or larger than $40 \mathrm{~mm}$ supplied larger amounts of adequate material for analysis than lesions with diameters of less than $40 \mathrm{~mm}$, and this difference was statistically significant.

In our study, we also observed that the location of the superior lobe lesions supplied a proportionally larger amount of adequate material for analysis when compared with other locations, and this difference was statistically significant. A possible explanation for this result is that it is often easier to reach the superior lobe injuries. Regardless of the position of the patient on the examination table, the punction needle generally reaches the injury at a straight angle. These lesions frequently have a pleural base and, consequently, a closefitting surface or even an infiltration of the chest wall, and this position favors the introduction of the needle to collect material for analysis without having to transpose the pleural space.

The patients who were referred due to suspicion of lung primary malignancy presented proportionally higher rates of adequate material for analysis in our study. One explanation for this finding could be the greater concern of the radiologist during the procedure for establishing a new cancer diagnosis rather than documenting a metastatic disease; however, this explanation is only an assumption.

Some lesion characteristics described in this study, such as the location on superior lobes and the suspicion of primary lung malignancy, were not described in previous studies. The only independent predictive factor of needle biopsy success was location in the superior lobes.

\section{CONCLUSION}

CT-guided percutaneous FNAB of lung lesions had a great rate of success in our study. Some radiologic characteristics of lung lesions may predict higher rates of success, such as a diameter equal to or larger than $40 \mathrm{~mm}$, suspicion of primary lung malignancy, and location in the superior lobes. These characteristics should be considered when ordering this procedure.

\section{REFERENCES}

1. Murphy JM, Gleeson FV, Flower CD. Percutaneous needle biopsy of the lung and its impact on patient management. World J Surg. 2001;25:3739.

2. Conces DJ Jr, Schwenk GR Jr, Doering PR, Glant MD. Thoracic needle biopsy. Improved results utilizing a team approach. Chest. 1987;91:8136.

3. Perlmutt LM, Johnston WW, Dunnick NR. Percutaneous transthoracic needle aspiration: A Review. Am J Roentgenol. 1989;152:451-5.

4. Westcott JL. Percutaneous transthoracic needle biopsy. Radiology. 1988;169:593-601.

5. Layfield LJ, Coogan A, Johnston WW, Patz EF. Transthoracic fine needle aspiration biopsy. Sensitivity in relation to guidance technique and lesion size and location. Acta Cytol. 1996;40:687-90.
6. Yankelevitz DF, Henschke CI, Koizumi JH, Altorki NK, Libby D. CTguided transthoracic needle biopsy of small solitary pulmonary nodules. Clin Imaging. 1997;21:107-10.

7. Miller JA, Pramanik BK, Lavenhar MA. Predicting the rates of success and complications of computed tomography-guided percutaneous coreneedle biopsies of the thorax from the findings of the preprocedure chest computed tomography scan. J Thorac Imaging. 1998;13:7-13.

8. Chojniak R, Isberner RK, Viana LM, Yu LS, Aita AA, Soares FA. Computed tomography guided needle biopsy: experience from 1,300 procedures. Sao Paulo Med J. 2006;124:10-4.

9. Weisbrod GL, Herman SJ, Tao LC. Preliminary experience with a dual cutting edge needle in thoracic percutaneous fine-needle aspiration biopsy. Radiology. 1987;163:75-8. 
10. Austin JH, Cohen MB. Value of having a cytopathologist present during percutaneous fine-needle aspiration biopsy of lung: report of 55 cancer patients and metaanalysis of the literature. Am J Roentgenol. 1993;160:175-7.

11. Milman N. Percutaneous lung biopsy with a fine bore cutting needle (Vacu-Cut): improved results using drill technique. Thorax. 1995;50:560-2.

12. Arakawa H, Nakajima $Y$, Kurihara $Y$, Niimi H, Ishikawa T. CT-guided transthoracic needle biopsy: a comparison between automated biopsy gun and fine needle aspiration. Clin Radiol. 1996;51:503-6.

13. Li H, Boiselle PM, Shepard JO, Trotman-Dickenson B, McLoud TC. Diagnostic accuracy and safety of CT-guided percutaneous needle aspiration biopsy of the lung: comparison of small and large pulmonary nodules. Am J Roentgenol. 1996;167:105-9.
14. Westcott JL. Direct percutaneous needle aspiration of localized pulmonary lesions: result in 422 patients. Radiology. 1980;137:31-5.

15. Stanley JH, Fish GD, Andriole JG, Gobien RP, Betsill WL, Laden SA, et al. Lung lesions: cytologic diagnosis by fine-needle biopsy. Radiology. $1987 ; 162: 389-91$

16. Khouri NF, Stitik FP, Erozan YS, Gupta PK, Kim WS, Scott WW Jr, et al. Transthoracic needle aspiration biopsy of benign and malignant lung lesions. Am J Roentgenol. 1985;144:281-8. 